\title{
Estudio descriptivo de los niños y adolescentes nuevos vistos en un servicio de salud mental en el periodo de un año
}

\section{A descriptive study of the child and adolescent new cases attended at a mental health service during one year}

\begin{abstract}
RESUMEN: En este trabajo se presenta un estudio descriptivo de los niños y adolescentes nuevos vistos en el Servicio de Salud Mental de Aranjuez (Área 11 de la Comunidad de Madrid) durante el año 2004. La muestra clínica consta de 314 pacientes nuevos. El propósito del estudio es doble: por un lado presentar algunas características sociodemográficas de los niños y adolescentes nuevos vistos y, por otro, mostrar los diagnósticos y las diferencias en función de la edad y el sexo. Se ha recurrido al programa informático de los Servicios de Salud Mental de distrito de la Comunidad de Madrid (Registro Acumulado de Casos: R. A. C.) para la obtención de los datos.

PALABRAS CLAVE: Servicios de Salud Mental, atención ambulatoria, epidemiología psiquiátrica, niños y adolescentes.
\end{abstract}

\begin{abstract}
We report a descriptive study of the children and adolescents attended for the first time at the Mental Health Services of Aranjuez (Area 11, Community of Madrid) during the year 2004. A total of 314 new patients were reviewed. The aim of this study is double: on one hand, to describe the socio-demographic characteristics of these new patients and, on the other, to study the distribution of the diagnostic categories with respect to sex and age variables. In order to collect the data, we used the computerised program of the Mental Health Services of the Community of Madrid (Accumulated Registry of Cases).

KEY WORDS: Mental Health Services, ambulatory care, psychiatric epidemiology, children and adolescents.
\end{abstract}

\section{Introducción.}

Existen aún problemas que dificultan el avance de la investigación sobre la epidemiología de los T. Psiquiátricos en niños y adolescentes (selección de la muestra, procedimientos utilizados-estructurados, semiestructurados, informadores...-, definición de caso, análisis de los datos, etc) y que explican la enorme variabilidad en los resultados de los estudios. Roberts y cols $^{32}$ realizaron una revisión de 52 estudios llevados a cabo en las décadas pasadas acerca de la prevalencia de psicopatología en niños y adolescentes y presentó unas estimaciones que oscilaban entre 1-51\%, con una prevalencia media de 15,8\%. En los trabajos internacionales se calcula una tasa de prevalencia entre el 10-25\% de los niños/as entre 0-18 años de edad de Trastornos Emocionales o de conducta que precisan/ podrían precisar una ayuda específica ${ }^{3124175}$. A pesar de esta gran variación en las tasas de prevalencia, se estima que al menos un $12 \%$ de los niños y adolescentes padecen trastornos mentales importantes, y que al menos la mitad de ellos se 
consideran seriamente incapacitados o alterados a causa de su enfermedad mental. Según un informe de la OMS se estima que aproximadamente el 5-15\% de la población infantil presenta trastornos psicológicos que afectan gravemente a la vida social del niño ${ }^{34}$. De acuerdo con los estudios epidemiológicos se ha llegado al consenso de que, en los países occidentales, en la infancia y la adolescencia, la prevalencia en tres meses a un año de los trastornos psiquiátricos está en torno al $15 \%$ y que un $7-8 \%$ tienen trastornos graves asociados con alguna dificultad en el funcionamiento psicosocial o educativo. Sin embargo, cuando se realizan evaluaciones repetidas en el tiempo, alrededor de un tercio de los individuos cumplen criterios para uno o más diagnósticos psiquiátricos en un momento u otro a mitad de la adolescencia ${ }^{5}$. De ellos, sólo una pequeña parte (inferior al 10\%) recibe una asistencia especializada $^{31}$. En torno al $30 \%$ de los niños/as que acuden al pediatra con quejas somáticas padecen trastornos emocionales. De ellos, sólo la mitad son identificados por el pediatra y menos de la mitad son derivados a un servicio específico ${ }^{31}$.

Las tasas de prevalencia varían en función de la edad, sexo y la clase social ${ }^{24}$ dependiendo de la muestra y los criterios diagnósticos utilizados ${ }^{17}$. El TDAH y los TC son las patologías más prevalentes en la mayoría de los casos, por encima de los T. Ansiedad y del estado de ánimo, aunque existe, sin embargo, disparidad en las prevalencias de los diferentes estudios ${ }^{18}$. La prevalencia de uno o más trastornos en la infancia y la adolescencia aumenta con la edad. En el trabajo de revisión de Roberts y cols ${ }^{1}$, las tasas de prevalencia media eran del 10,2\% en los niños en edad preescolar, del 13,2\% en los preadolescentes y del 16,5\% en los adolescentes $^{34}$. El sexo o género, aunque con una gran confusión terminológica y conceptual ${ }^{16}$, constituye también una variable importante y relevante que hay que tener en cuenta en la presentación de psicopatología en niños y adolescentes. Hay cuadros que se presentan con predominancia en niños (trastornos de inicio temprano que implican deterioro del neurodesarrollo: dislexia/retraso de la lectura, trastornos del espectro autista, TDAH, Trastorno antisocial persistente de inicio temprano) y otros son más frecuentes en niñas: trastornos emocionales de inicio en la adolescencia (depresión, trastornos de la conducta alimentaria, trastornos por. Ansiedad) ${ }^{33}$. Por otra parte, el TDAH y la ansiedad de separación disminuyen con la edad, mientras que el trastorno negativista desafiante es más prevalente entre adolescentes, el trastorno por estrés postraumático es mayor entre los adolescentes que en niños y la depresión mayor aumenta con la edad ${ }^{18}$.

En España, las prevalencias parecen ser similares a las de otros países ${ }^{18}$. Las tasas medias estimadas de trastorno mental en la infancia y adolescencia en España son: Incidencia (anual, administrativa): 7-8 por mil habitantes menores de 15 años; Prevalencia (anual, administrativa): 12-14 por mil habitantes menores de 15 años; Frecuentación (anual): 80-90 contactos/año por mil habitantes menores de 15 años $^{31}$. 
Los trastornos psiquiátricos en la infancia y la adolescencia constituyen, por tanto, una de las causas más importantes de morbilidad (y mortalidad) y un gran problema de Salud Pública

Existen escasos estudios de epidemiología en psiquiatría infanto-juvenil en España ${ }^{18}$. Son necesarios más estudios y debería constituir una línea básica de investigación ${ }^{34}$.

A diferencia de los estudios realizados en muestras comunitarias, las tasas de prevalencia e incidencia de los trastornos psiquiátricos en muestras clínicas aportan información sobre la demanda real que se produce en un determinado servicio. Este dato representa un indicador fiable en la optimización de la gestión y planificación de los recursos disponibles ${ }^{15}$. Creemos que los datos clínicos que hemos obtenido analizando la actividad asistencial infanto - juvenil de todo un año en el Servicio de Salud Mental del distrito de Aranjuez es un buen ejemplo de ello.

El Servicio de Salud Mental que ha venido atendiendo a la población del distrito sanitario de Aranjuez (Madrid) desde 1986 es fruto de la colaboración entre la Comunidad de Madrid y la Congregación de Hermanas Hospitalarias del Sagrado Corazón de Jesús (Complejo Asistencial Benito Menni de Ciempozuelos). Esta colaboración ha adoptado varias modalidades administrativas a lo largo del tiempo, siendo la última la modalidad de concierto público. Esta situación se ha mantenido hasta la finalización del último concierto, en diciembre de 2005.

El Servicio de Salud Mental de Aranjuez atendía la población del distrito sanitario de Aranjuez, incluida en el Área sanitaria número 11 de la Comunidad Autónoma de Madrid. Como se observa en la figura 1, esta área está formada por dos sectores geográficos muy diferenciados. Existe un territorio de carácter urbano, situado en el sur del municipio de Madrid. El segundo sector comprende el distrito sanitario de Aranjuez propiamente dicho y engloba las comarcas de las vegas de los ríos Jarama, Tajo y Tajuña y está compuesto por un conjunto de municipios de carácter semiurbano y rural. La población de este distrito ascendía a 115.471 habitantes según el último censo de 2001, cifra que en el año 2004 ha sido claramente superada (a 30 de abril de 2005 el número de tarjetas individuales sanitarias, según datos de la Gerencia de Atención Primaria del Área 11 se sitúa en torno a 150000). En la tabla 1 se recogen las principales características sociodemográficas de este distrito y su comparativa con respecto al total de población de la Comunidad de Madrid ${ }^{21}$. 
FIGURA 1: ÁREA 11 Y DISTRITO DE ARANJUEZ (MEMORIA A. P. 2003)

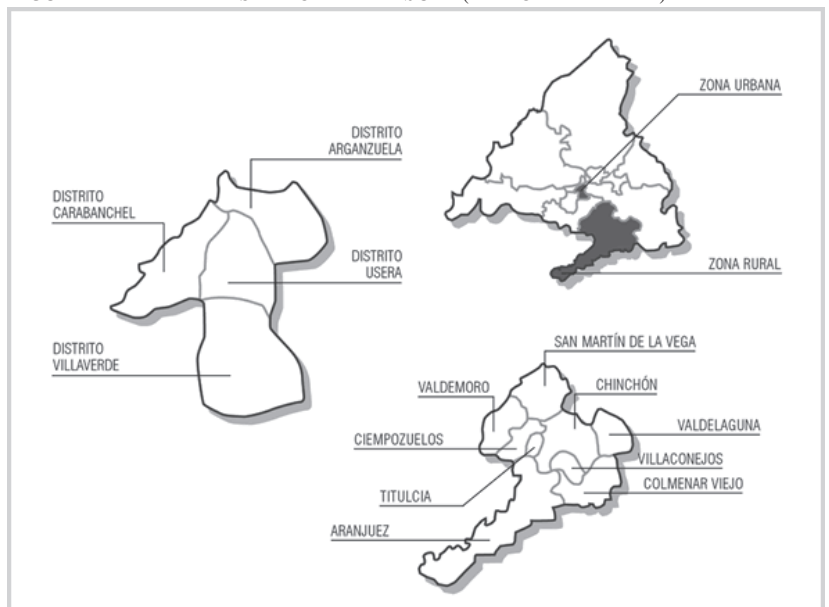

Tabla 1

Datos sociodemográficos población Distrito - Comunidad de Madrid

\begin{tabular}{lcc}
\hline & DISTRITO ARANJUEZ & COMUNIDAD DE MADRID \\
\hline $\mathrm{N}^{\mathrm{o}}$ habitantes (2004) & 130.012 & 5.964 .143 \\
\hline Varones (\%) & 50,08 & 48,42 \\
\hline Mujeres (\%) & 49,9 & 51,57 \\
\hline Edad (\%) (Censo 2001) & 17,51 & 14,29 \\
\hline$<15$ años & 69,91 & 71,15 \\
\hline 15 -64 años & 12,57 & 14,55 \\
\hline$>$ 64 años & 3,31 & 3,46 \\
\hline Desempleo (\%) & 1,79 & 1,40 \\
\hline Ratio desempleo Mujer / varón & & 0,80 \\
\hline Actividad profesional (\%) & 5,54 & 13,51 \\
\hline Agrario & 19,93 & 9,61 \\
\hline Industria & 18,37 & 76,08 \\
\hline Construcción & 56,14 & $13.094,11$ \\
\hline Servicios & $9.047,76$ & 20,74 \\
\hline Renta Per cápita ( $€$ ) & & 50,09 \\
\hline Nivel educativo (\%) & 7,59 & 17,44 \\
\hline Tercer grado & 48,26 & 9,97 \\
\hline Segundo grado & 24,29 & 1,77 \\
\hline Primer grado & 16,04 & \\
\hline Sin estudios & 3,79 & \\
\hline Analfabeta & & \\
\hline
\end{tabular}


ORIGINALES Y REVISIONES

Se trata de un distrito semirural, salvo el municipio de Valdemoro que se puede considerar incluido dentro del área de influencia de Madrid, y el municipio de Aranjuez, caracterizado por una actividad volcada en el sector servicios. Es una población más joven que el resto de la Comunidad de Madrid. El nivel socioeconómico está por debajo del promedio de la Comunidad de Madrid. El nivel educativo es también inferior, con mayor proporción de analfabetismo y carencia de estudios básicos. La proporción de población con estudios superiores es escasa en comparación con el resto del territorio de la Comunidad.

Es un distrito de una gran extensión y dispersión. Algunos municipios distan hasta $25-30 \mathrm{Km}$. del punto asistencial más cercano del servicio, con una mala oferta de transporte público. Por este motivo, de cara a mejorar la accesibilidad de la población, la actividad asistencial del Servicio se llevaba a cabo en tres dispositivos asistenciales: Aranjuez (dispositivo principal y cabecera del distrito), Valdemoro y Ciempozuelos. Cada una de estas sedes atendía a su población de referencia de acuerdo al siguiente esquema:

Dispositivo principal (Aranjuez): Atendía dos Zonas Básicas de Salud (Aranjuez y Colmenar de Oreja), con tres Equipos de Atención Primaria (Aranjuez I y II, Colmenar de Oreja) cubriendo los siguientes municipios: Aranjuez, Colmenar de Oreja, Chinchón, Valdelaguna, Villaconejos.

Dispositivo de Valdemoro: Atendía a la Zona Básica de Salud de Valdemoro, prestando asistencia a la población del municipio de Valdemoro, que incluye dos Equipos de Atención Primaria.

Dispositivo de Ciempozuelos: Atendía dos Zonas Básicas de Salud (Ciempozuelos y San Martín de la Vega) cubriendo los municipios de Ciempozuelos, Titulcia y San Martín de la Vega. Incluye dos Equipos de Atención Primaria.

La atención a la población infanto - juvenil se realizaba sólo en dos dispositivos. En Aranjuez se atendían a los niños y adolescentes de Aranjuez, Zonas Básicas de Colmenar de Oreja, Ciempozuelos y San Martín de la Vega. En Valdemoro se atendía exclusivamente a la población de dicho municipio.

La Comunidad de Madrid está organizada, sanitariamente, en once áreas de salud, cada una de estas áreas se subdivide en varios distritos sanitarios. Cada distrito consta de un Servicio de Salud Mental, que se encarga de la atención ambulatoria de todos los problemas psiquiátricos y de salud mental de toda la población de referencia. El funcionamiento de los Servicios de Salud Mental es el propio de la Atención Especializada, siendo su principal ruta asistencial la procedente de Atención Primaria, si bien es posible la derivación de casos desde los diferentes especialistas, atención hospitalaria, así como otras instancias no sanitarias (Servicios Sociales, Equipos de Orientación Psicopedagógicos, Administración de Justicia, etc.).

El modelo que fundamenta estos Servicios de Salud Mental es el de la Psi- 
quiatría Comunitaria ${ }^{14}$. Los Servicios están constituidos por equipos multidisciplinares, integrados por psiquiatras, psicólogo/as clínicos, trabajador/as sociales, enfermero/a y administrativo/a. En estos Servicios se actúa mediante programas de obligada implantación por la Comunidad de Madrid ${ }^{11}$. Estos programas cubrirían las diferentes necesidades asistenciales detectadas en un determinado territorio: patología infantil y juvenil, atención ambulatoria, psicogeriatría, rehabilitación, alcoholismo, etc.

Los diferentes Servicios de Salud Mental actúan en coordinación con una red de dispositivos asistenciales de Salud Mental (dispositivos de rehabilitación, hospitalización y socio comunitarios) localizados en cada una de las áreas sanitarias. Esta distribución no está al mismo nivel en todas las áreas de salud, habiéndose producido un importante desarrollo en estos últimos años en el Area 11. En el Servicio de Salud Mental de Aranjuez se contaba, en el año 2004, con los siguientes dispositivos:

Dispositivos de Rehabilitación: Centros de Rehabilitación Psicosocial "Los Cármenes" y "Nuestra Señora del Sagrario"; Centro de Rehabilitación Laboral; Mini- Residencia "Carabanchel". Estos dispositivos se sitúan en el Municipio de Madrid. Unidades de Media y Larga Estancia (Complejo Asistencial Benito Menni y Hospital San Juan de Dios) situados en el municipio de Ciempozuelos; Centros de Día de Aranjuez, Valdemoro y Mancomunidad de la Vega (Ciempozuelos). A finales del año 2004 comenzó a funcionar un Centro de Rehabilitación Psicosocial y un Centro de Día en el Municipio de Aranjuez.

Dispositivos de soporte social: Pisos supervisados, situados en el municipio de Madrid.

Otros recursos de soporte social: Parroquias, O. N. G., Asociaciones, etc.

Recursos socio comunitario: son compartidos con el resto de la población (recursos educativos, residenciales, de empleo, etc.)

Dispositivos de hospitalización: En el caso de la población adulta, las Unidades de Hospitalización Breve de referencia son las ubicadas en el H. U. "12 de Octubre" (Madrid) y el "Complejo Asistencial Benito Menni" (Ciempozuelos). Para la población infanto - juvenil los hospitales de referencia son Hospital "Niño Jesús" (Madrid) y la Unidad de Adolescentes del H. G. U. Gregorio Marañón (Madrid). Unidad de Deshabituación alcohólica (Situada al Norte del Municipio de Madrid).

Centro Comarcal de Drogodependencias (Aranjuez) y Agencia Antidroga: La sectorización de la Comunidad de Madrid establece que la patología por drogodependencia es atendida en una ruta asistencial diferente a Salud Mental, excepto en los casos de Alcoholismo. Esta distinción es difícil de mantener en la práctica diaria de ambas redes.

Otros dispositivos, como los hospitales de día, se han ido desarrollando a 
partir del 2004. Con anterioridad estaban disponibles los situados en otras áreas de Madrid, geográficamente muy inaccesibles para nuestra población. En el caso de la población infanto - juvenil los hospitales de día disponibles en el 2004 eran el Hospital de Día de Herrera Oria (Norte de Madrid) y el Hospital de Día de la Pradera de San Isidro (distrito de Carabanchel), en el Municipio de Madrid.

Respecto a los recursos humanos del propio Servicio de Salud Mental, en el año 2004 el equipo estaba constituido por cinco psiquiatras, dos psicólogos a tiempo completo y uno a tiempo parcial, dos enfermeras, una trabajadora social, dos administrativas a tiempo completo y una a tiempo parcial. Para la atención a la población infanto - juvenil del distrito (hasta los 18 años), de los recursos mencionados, se destinaban un psiquiatra (simultaneando tareas de coordinación como responsable del Servicio) y una psicóloga clínica a tiempo completo como subequipo específico. El resto de actuaciones profesionales (enfermería, trabajo social, administración, etc.) eran compartidas con el resto de programas y tareas asistenciales. La atención infanto - juvenil, realizada en los dispositivos, se completaba con las actividades de coordinación con todas las instancias sanitarias, educativas y sociales necesarias.

La actividad asistencial global del Servicio e indicadores, durante el año 2004, figura en la tabla 2. En esta tabla se detallan también las cifras específicas de la población hasta quince años, precisamente las que proporciona el programa informático de la Comunidad de Madrid.

Tabla 2

Indicadores de actividad asistencial del SSM Aranjuez en el año 2004

\begin{tabular}{|c|c|c|c|c|c|c|c|c|c|}
\hline & \multicolumn{4}{|c|}{ HOMBRES } & \multicolumn{3}{|c|}{ MUJERES } & \multicolumn{2}{|c|}{ TOTAL } \\
\hline & $<16$ & $>64$ & Total & $<16$ & $>64$ & Total & $<16$ & $>64$ & Total \\
\hline $\begin{array}{l}\text { Población } \\
\text { (Censo 2001) }\end{array}$ & 11.087 & 6.098 & 57.350 & 10.500 & 8.418 & 58.121 & 21.587 & 14.516 & 115.471 \\
\hline Pacientes En Contacto & 345 & 129 & 1.497 & 217 & 373 & 2.333 & 562 & 502 & 3.830 \\
\hline Casos Nuevos & 185 & 33 & 594 & 141 & 80 & 809 & 326 & 113 & 1.403 \\
\hline Asistencias Totales & 1.584 & 469 & 8255 & 778 & 1.413 & 11.776 & 2.362 & 1.882 & 20.031 \\
\hline $\begin{array}{l}\text { Incidencia } \\
\text { Administrativa }\end{array}$ & 16,69 & 5,41 & 10,36 & 13,43 & 9,50 & 13,92 & 15,10 & 7,78 & 12,15 \\
\hline $\begin{array}{l}\text { Frecuentación } \\
\text { Administrativa }\end{array}$ & 117,71 & 64,12 & 120,00 & 61,05 & 136,97 & 167,07 & 90,15 & 106,37 & 143,69 \\
\hline $\begin{array}{l}\text { Prevalencia } \\
\text { Administrativa }\end{array}$ & 31,12 & 21,15 & 26,10 & 20,67 & 44,31 & 40,14 & 26,03 & 34,58 & 33,17 \\
\hline
\end{tabular}


Durante el año 2004 se ha producido nuevamente un incremento en el número de pacientes nuevos, pacientes en contacto e indicadores de actividad asistencial, respecto a los años anteriores, como se puede apreciar en la figura 2.

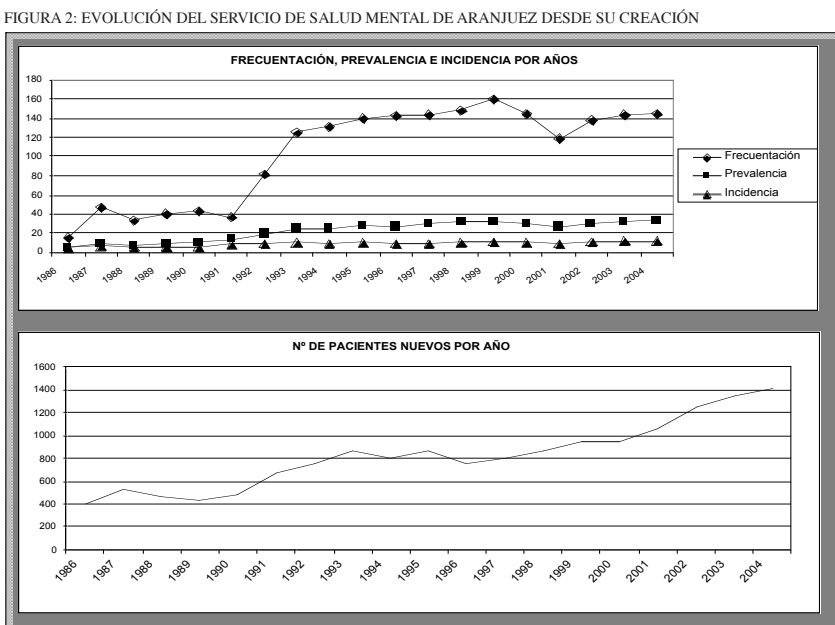

En la figura 3 se indica la evolución de la actividad asistencial referida a la población infanto-juvenil del SSM de Aranjuez en los últimos años.

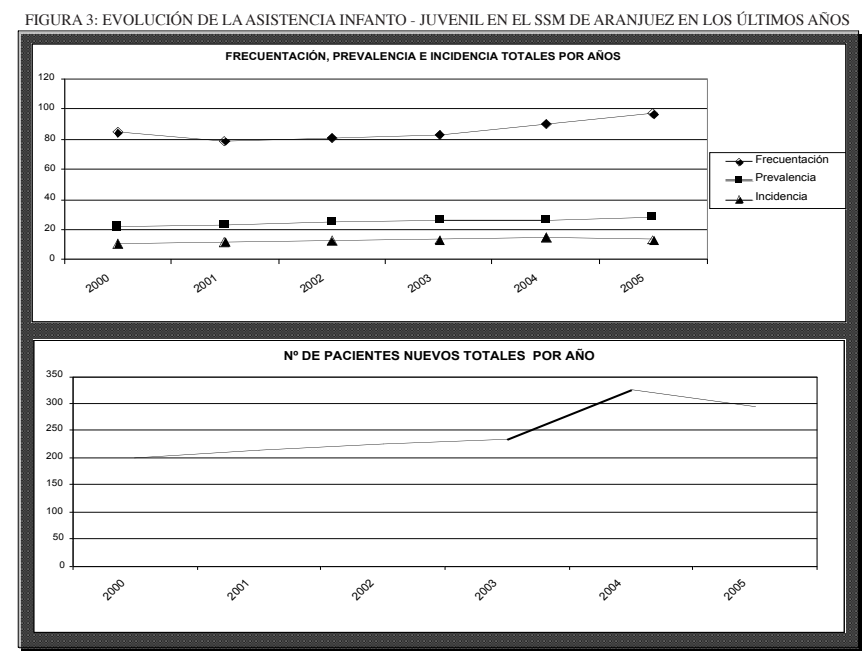


ORIGINALES Y REVISIONES

En el año 2004 tuvo lugar el atentado terrorista conocido como 11-M. El nivel de afectación sobre este distrito fue muy bajo. Los datos reflejados en este trabajo no incluyen los casos diagnósticos catalogados como afectados por el 11-M.

Estos indicadores son generados por el programa de Registro Acumulado de Casos. La incidencia recoge el número de casos nuevos por mil habitantes; respecto a la prevalencia, consiste en el número de casos nuevos y antiguos por mil habitantes en contacto con el servicio. La frecuentación señala el número de consultas realizadas por mil habitantes. La actualización de las cifras poblacionales modifica estos indicadores, circunstancia que se produce coincidiendo con los censos oficiales (último en el año 2001). A pesar de este ajuste corrector, como se puede observar en la figura 2 , se aprecia un constante incremento desde la creación del Servicio, en consonancia con las características demográficas del distrito (zona de la Comunidad de Madrid en expansión urbanística, receptora de nueva población).

Con posterioridad a la realización de este trabajo, el Servicio de Salud Mental del distrito sanitario de Aranjuez ha experimentado notables cambios. En el año 2006 la población del municipio de Valdemoro comenzó a ser atendida por un Servicio de Salud Mental independiente y de gestión pública dependiente del Hospital 12 de Octubre, pero desde el año 2008 esta población es atendida por la empresa Capio que, a su vez, ha acordado su gestión con el Hospital San Juan de Dios de Ciempozuelos. El resto del distrito sanitario sigue siendo atendido bajo gestión privada (modalidad de concierto) pero por dos empresas diferentes: el dispositivo de Ciempozuelos continúa a cargo del Complejo Asistencial Benito Menni (Hermanas Hospitalarias del Sagrado Corazón de Jesús) y el dispositivo de Aranjuez es gestionado por el Hospital San Juan de Dios de Ciempozuelos (Hermanos de San Juan de Dios).

Este trabajo pretende la descripción de los niños y adolescentes nuevos vistos en el Servicio de Salud Mental de Aranjuez durante el año 2004, sus diagnósticos y las diferencias en función de la edad y el género.

\section{Material y Métodos.}

Los pacientes, objeto de este estudio, son derivados fundamentalmente desde los Equipos de Atención Primaria del distrito y, en menor medida, de Atención Especializada. Es importante señalar que los problemas relacionados con el consumo de tóxicos, con la excepción del alcohol, tienen una ruta asistencial diferenciada. Son remitidos mayoritariamente desde Atención Primaria al Centro 
Comarcal de Drogodependencias, situado en Aranjuez. Hay una parte importante de psicopatología relacionada con el alcohol (patología dual) que es probable que no sea vista en este SSM, dado el patrón habitual de policonsumo.

En el caso de la atención infanto - juvenil una pequeña parte de demandas procedían de las instancias educativas y servicios sociales.

El SSM de Aranjuez, como todos los SSM de distrito de la Comunidad de Madrid cuenta con un programa informático para el registro de datos sociodemográficos, diagnósticos y asistenciales que permite el seguimiento y control de la actividad del Servicio. Este programa se conoce como Registro Acumulado de Casos Psiquiátricos (RAC); es proporcionado y controlado por la Oficina Regional de Coordinación de Salud Mental.

Los datos que se introducen en este RAC proceden de varias fuentes:

Hoja de Identificación del Paciente: Se cumplimenta en el momento de la recepción del paciente en el Servicio e incluye los siguientes datos: identificación del paciente, domicilio, estado civil, tipo de convivencia, nivel educativo, procedencia, profesión, situación laboral y, en el caso de niños y adolescentes, escolarización y datos del padre /madre. Este protocolo es cumplimentado por personal administrativo y se lleva a cabo en el mostrador de recepción, con escasa intimidad en la transmisión de los datos, que es realizada de forma oral.

Ficha de Asistencia: Se cumplimenta tras cada acto asistencial con el paciente, recogiendo los siguientes datos: tipo de prestación recibida, modalidad de atención, grupo de edad y diagnósticos según la codificación de la novena edición de la Clasificación Internacional de Enfermedades de la Organización Mundial de la Salud ${ }^{30}$. Este protocolo es realizado por los profesionales clínicos (Psiquiatras y psicólogos clínicos).

Este programa informático proporciona una serie limitada de listados para el análisis de la actividad asistencial. Estos listados han constituido la base para la elaboración de este trabajo, no siendo posible la obtención de todas las combinaciones de variables posibles. En el caso concreto del programa infanto - juvenil, la estadística básica que proporciona se refiere solamente al grupo de población de cero a quince años, cuando la actividad asistencial para este programa alcanza a los dieciocho años. Esto constituye una limitación importante para obtener resultados, conclusiones y análisis.

Para la realización de este trabajo se ha recurrido a un listado auxiliar del RAC que permite obtener datos de pacientes nuevos entre dieciséis y dieciocho años. Por otra parte, el programa informático proporciona cifras ligeramente dispares según el tipo de listado solicitado o variables como la fecha de la solicitud de la información. Esto explica las variaciones que se muestran en este estudio, por ejemplo, en el número de casos nuevos en función de la edad.

De acuerdo con este programa, se considera paciente nuevo a toda persona 
que nunca antes había tenido contacto con el Servicio, por lo que quedan excluidos de la consideración de nuevos aquellas personas vistas durante el 2004 que tuvieran un contacto previo. Estos casos no son considerados como nuevos por el programa, aunque hayan pasado años.

El análisis de este trabajo se centra en los pacientes nuevos, menores de 18 años, vistos en el año 2004 según la consideración de este programa informático, y atendiendo al primer diagnóstico codificado en la primera consulta. La falta de un diagnóstico definido en la primera consulta se consignaba como diagnóstico sin especificar.

Las categorías diagnósticas consideradas han sido los epígrafes generales de la Novena Edición de la Clasificación Internacional de Enfermedades de la Organización Mundial de la Salud 30. Esta novena edición (1977) es la que aún permanece vigente en la Red de Salud Mental de la Comunidad de Madrid. El conjunto de categorías diagnósticas empleadas puede consultarse en la tabla 3. En algunas categorías se han desglosado los datos por que entendemos que ofrecen un mejor análisis, por ejemplo: se ha convertido al diagnóstico de Abuso de alcohol en una categoría principal (305.0), se ha desglosado la categoría de síndromes especiales no clasificados en otra parte (307) y se han unificado las diferentes categorías de retraso mental.

Tabla 3

Categorías diagnósticas principales C.I.E.-9-O. M.S.

\begin{tabular}{ll}
\hline 290 & Psicosis orgánica senil y presenil \\
\hline 291 & Psicosis alcohólicas \\
\hline 292 & Psicosis debidas a drogas \\
\hline 293 & Psicosis orgánicas transitorias \\
\hline 294 & Otras psicosis orgánicas (crónicas) \\
\hline 295 & Psicosis esquizofrénica \\
\hline 296 & Psicosis afectivas \\
\hline 297 & Estados paranoides \\
\hline 298 & Otras psicosis no orgánicas \\
\hline 299 & Psicosis peculiares de la niñez \\
\hline 300 & Trastornos neuróticos \\
\hline 301 & Trastornos de la personalidad \\
\hline 302 & Desviaciones y trastornos sexuales \\
\hline 303 & Síndrome de dependencia del alcohol \\
\hline 304 & Dependencia de la drogas \\
\hline 3050 & Alcohol (abuso) \\
\hline 305 & Abuso de drogas sin dependencia \\
\hline 306 & Alteraciones corporales originadas por factores mentales \\
\hline 307 & Síntomas o síndromes especiales no clasificados en otra parte \\
& Movimiento \\
& Alimentación \\
& Sueño \\
& Esfínteres \\
& Dolor
\end{tabular}




\begin{tabular}{ll}
308 & Reacción aguda ante gran tensión \\
\hline 309 & Reacción de adaptación \\
\hline 310 & Trastornos mentales no psicóticos por lesión orgánica encefálica \\
\hline 311 & Trastornos depresivos no clasificados otra parte \\
\hline 312 & Perturbación conducta no clasificada otra parte \\
\hline 313 & Perturbación de las emociones propias de la niñez y adolescencia \\
\hline 314 & Síndrome hipercinético de la niñez \\
\hline 315 & Retardo selectivo del desarrollo \\
\hline 316 & $\begin{array}{l}\text { Factores psíquicos asociados con enfermedades clasificadas en otra parte } \\
\text { Retraso mental } \\
\text { Sin especificar } \\
\text { No patología } \\
\text { Códigos V }\end{array}$ \\
&
\end{tabular}

\section{Resultados.}

Las características de la muestra clínica vienen recogidas en la tabla 4. Respecto del total de pacientes nuevos vistos en el SSM de Aranjuez en el año 2004, el grupo de cero a quince años constituye el 24,7 \%. La mayor parte de niños y adolescentes viven con ambos padres $(77,4 \%)$. En el caso de vivir sólo con un progenitor, este es fundamentalmente la madre y sólo en un 0,9 \% vive con el padre.

Tabla 4

Datos de la muestra clínica

HOMBRES INFANTO - JUVENIL

\begin{tabular}{lr}
\hline $\mathrm{N}^{\mathrm{o}}$ Nuevos & 173 \\
\hline Edad media & 9,02 \\
\hline VAR edad & 12,3 \\
\hline MED edad & 9 \\
\hline MODA edad & 7 \\
\hline PER 75 edad & 12 \\
\hline PER 25 edad & 6 \\
\hline
\end{tabular}

MUJERES INFANTO - JUVENIL

\begin{tabular}{lc}
\hline $\mathrm{N}^{\circ}$ Nuevos & 141 \\
\hline Edad media & 9,75 \\
\hline VAR edad & 15,6 \\
\hline MED edad & 10 \\
\hline MODA edad & 15 \\
\hline PER 75 edad & 13 \\
\hline PER 25 edad & 6 \\
\hline
\end{tabular}

CONVIVENCIA MENORES (hasta 16 años)\%

\begin{tabular}{lr} 
Con ambos padres & 77,4 \\
\hline Sólo con madre & 20,9 \\
\hline Sólo con padre & 0,9 \\
\hline Con otros familiares & 0,6 \\
\hline En institución & 0,3 \\
\hline
\end{tabular}

TOTAL DE NUEVOS POR EDADES

\begin{tabular}{lr}
\hline $31-45$ & 30,6 \\
\hline $0-15$ & 24,7 \\
\hline $16-30$ & 21,8 \\
\hline $46-60$ & 14,1 \\
\hline $61-75$ & 6 \\
\hline $75-$ & 3,3 \\
\hline & \\
TOTAL INFANTO JUVENIL & 314 \\
\hline$N^{o}$ Nuevos & 9,35 \\
\hline Edad media
\end{tabular}


El número total de casos nuevos, incluyendo todos los menores de dieciocho años, asciende a 314. La distribución por sexos muestra una mayoría de varones $(55,09 \%$ / 44,90\%). La edad media de la muestra es de 9,35 años. Si distinguimos sexos, la media, varianza, mediana y amplitud intercuartil de la edad en las mujeres es ligeramente mayor.

Las modas de la edad son muy dispares en función del sexo, siendo siete años el valor en los varones y quince en las mujeres. a año.

En la figura 4 se refleja el número de pacientes nuevos por sexo y edad, año

Figura 4

Número de pacientes nuevos en función del sexo y la edad

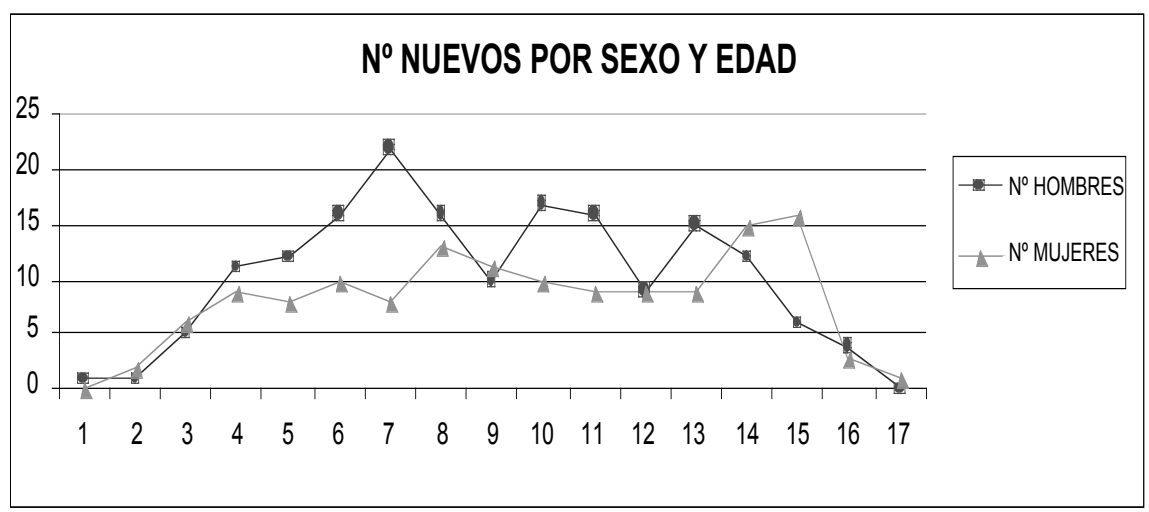

En cuanto a los diagnósticos aplicados en la primera consulta, incluidos todos los menores de dieciocho años, los resultados se ofrecen en función de la edad en la tabla 5 , en función del sexo en la tabla 6 y combinando ambas variables en las tablas 7, 8 y 9 . Cuando nos referimos a la variable edad se consideran los siguientes intervalos de edad: 0-6, 7-12 y 13-17. 
Tabla 5

Diagnósticos en función de la edad (\%)

\begin{tabular}{llccc}
\hline CÓDIGO & Diagnóstico & 0,6 & $7-12$ & $13-17$ \\
\hline 296 & Psicosis afectivas & - & - & 1.23 \\
\hline 299 & Psicosis peculiares de la niñez & 1.23 & - & - \\
\hline 300 & Trastornos neuróticos & 1.23 & 5.26 & 6.17 \\
\hline 301 & Trastornos de la personalidad & - & - & 1.23 \\
\hline 303 & Síndrome de dependencia del alcohol & - & - & 1.23 \\
\hline 304 & Dependencia de la drogas & - & - & 1.23 \\
\hline 305 & Abuso de drogas sin dependencia & - & - & 1.23 \\
\hline 306 & Alteraciones corporales, orig. Por factores mentales & - & - & 2.46 \\
\hline & Movimiento & - & 1.31 & 3.7 \\
\cline { 2 - 5 } & Alimentación & 7.4 & 0.65 & 7.4 \\
\hline 307.4 & Sueño & 6.17 & 1.31 & 3.7 \\
\cline { 2 - 4 } & Esfínteres & 8.64 & 7.89 & - \\
\hline Dolor & Reacción aguda ante gran tensión & - & 0.65 & - \\
\hline 308 & Reacción de adaptación & 2.46 & 1.31 & - \\
\hline 309 & Trastornos depresivos no clasificados en otra parte & 11.11 & 26.97 & 27.16 \\
\hline 311 & Perturbación conducta no clasificada en otra parte & - & - & 1.23 \\
\hline 312 & Perturb. Emociones propias niñez y adolescencia & 3.7 & 1.31 & 8.64 \\
\hline 313 & Síndrome hipercinético de la niñez & 23.45 & 19.07 & 11.11 \\
\hline 314 & Retardo selectivo del desarrollo & 8.64 & 13.81 & 4.93 \\
\hline 315 & Retraso mental & 1.23 & 1.31 & 1.23 \\
\hline & Sin especificar & 1.23 & 0.65 & - \\
\hline No patología & 16.04 & 14.47 & 11.11 \\
\hline & & 7.4 & 3.94 & 4.93 \\
\hline
\end{tabular}

Tabla 6

Diagnósticos en función del sexo (\%)

\begin{tabular}{llcl}
\hline CÓDIGO & Diagnóstico & Varones & Mujeres \\
\hline 296 & Psicosis afectivas & 0.58 & - \\
\hline 298 & Otras psicosis no orgánicas & 0.58 & - \\
\hline 299 & Psicosis peculiares de la niñez & 0.58 & - \\
\hline 300 & T. Neuróticos & 2.89 & 6.38 \\
\hline 301 & T. Personalidad & 0.58 & - \\
\hline 303 & Dependencia alcohol & 0.58 & - \\
\hline 304 & Dependencia drogas & - & 0.71 \\
\hline 305 & Abuso drogas & - & 0.71 \\
\hline 306 & Alt. Corporales por factores mentales & 0.58 & - \\
\hline & Movimiento & 2.89 & - \\
307.4 & Sueño & 2.31 & 4.26 \\
\hline & Alimentación & 2.89 & 5.67 \\
\cline { 2 - 4 } & Esfínteres & 5.78 & 6.38 \\
\hline 307.9 & Otros y los no esp. & - & 0.71 \\
\hline 308 & R. Aguda & 0.58 & 2.13 \\
\hline 309 & R. Adaptación & 24.28 & 21.28 \\
\hline 311 & T. Depresivo no clas. & - & 0.71 \\
\hline 312 & P. Conducta no clasificada en otra parte & 1.73 & 6.38 \\
\hline 313 & P. Emociones propias niñez y adolescencia & 16.76 & 19.86 \\
\hline 314 & S. Hipercinético & 15.03 & 4.26 \\
\hline 315 & Retardo selectivo desarrollo & 0.58 & 2.13 \\
\hline & Retraso mental & 1.16 & - \\
\hline & Sin especificar & 16.76 & 10.64 \\
\cline { 2 - 3 } & No patología & 2.80 & 7.80 \\
\hline & & &
\end{tabular}




\section{ORIGINALES Y REVISIONES}

Tabla 7

Diagnósticos en función de la edad y el sexo (\%)

0 a 6 años

\begin{tabular}{llcc}
\hline CÓDIGO & Diagnóstico & Varones & Mujeres \\
\hline 299 & Psicosis peculiares de la niñez & 2.17 & - \\
\hline 300 & Trastornos neuróticos & - & 2.85 \\
\hline & Alimentación & 8.69 & 5.71 \\
\cline { 2 - 4 } & Sueño & 6.52 & 5.71 \\
\cline { 2 - 5 } & Esfínteres & 6.52 & 11.42 \\
\hline 308 & Reacción aguda ante gran tensión & - & 5.71 \\
\hline 309 & Reacción adaptación & 8.69 & 14.28 \\
\hline 312 & Perturbación de la conducta no clasificada en otra parte & - & 8.57 \\
\hline 313 & Perturb. Emociones propias niñez y adolescencia & 28.26 & 17.14 \\
\hline 314 & Síndrome hipercinético & 15.21 & - \\
\hline 315 & Retardo selectivo del desarrollo & 2.17 & - \\
\hline & Retraso mental & 2.17 & - \\
\cline { 2 - 4 } & Sin especificar & 15.21 & 17.14 \\
\cline { 2 - 4 } & No patología & 4.34 & 11.42 \\
\hline
\end{tabular}

Tabla 8

Diagnósticos en función de la edad y el sexo (\%)

7 a 12 años

\begin{tabular}{llcc}
\hline CÓDIGO & Diagnóstico & Varones & Mujeres \\
\hline \multirow{2}{*}{300} & Trastornos neuróticos & 4.44 & 6.45 \\
\hline & Movimiento & 2.22 & - \\
\cline { 2 - 4 } & Alimentación & 1.11 & - \\
\cline { 2 - 4 } & Sueño & - & 3.22 \\
\cline { 2 - 4 } & Esfinteres & 7.77 & 8.06 \\
\cline { 2 - 4 } & Dolor & - & 1.61 \\
\hline 308 & Reacción aguda ante gran tensión & 1.11 & 1.61 \\
\hline 309 & Reacción de adaptación & 27.77 & 25.8 \\
\hline 312 & Perturbación de la conducta no clasificada en otra parte & - & 3.22 \\
\hline 313 & Perturbación de las emociones propias de la niñez y adolescencia & 16.66 & 22.58 \\
\hline 314 & Síndrome hipercinético de la niñez & 18.88 & 6.45 \\
\hline 315 & Retardo selectivo del desarrollo & - & 3.22 \\
\cline { 2 - 4 } & Retraso mental & 1.11 & - \\
\cline { 2 - 4 } & Sin especificar & 17.77 & 9.67 \\
\cline { 2 - 4 } & No patología & 1.11 & 8.06 \\
\hline
\end{tabular}


Tabla 9

Diagnósticos en función de la edad y el sexo (\%)

13 a 17 años

\begin{tabular}{llcc}
\hline CÓDIGO & Diagnóstico & Varones & Mujeres \\
\hline 296 & Psicosis afectivas & 2.7 & - \\
\hline 300 & Trastornos neuróticos & 2.7 & 9.09 \\
\hline 301 & Trastornos de personalidad & 2.7 & - \\
\hline 303 & Dependencia alcohol & 2.7 & - \\
\hline 304 & Dependencia drogas & - & 2.27 \\
\hline 305 & Abuso drogas & - & 2.27 \\
\hline 306 & Alter. Corp. Orig. Factores mentales & 5.4 & - \\
\hline & Movimiento & 8.1 & - \\
\cline { 2 - 4 } & Alimentación & - & 13.63 \\
\cline { 2 - 4 } & Sueño & 2.7 & 4.54 \\
\hline 309 & Reacción de adaptación & 35.13 & 20.45 \\
\hline 311 & Trast. Depresivos no clasificados & - & 2.27 \\
\hline 312 & Perturbación de la conducta no clasificada en otra parte & 8.1 & 9.09 \\
\hline 313 & Perturbación de las emociones propias de la niñez y adolescencia & 2.7 & 18.18 \\
\hline 314 & Síndrome hipercinético de la niñez & 5.4 & 4.54 \\
\hline 315 & Retardo selectivo del desarrollo & - & 2.27 \\
\hline & Sin especificar & 16.21 & 6.81 \\
\cline { 2 - 4 } & No patología & 5.4 & 4.54 \\
\hline
\end{tabular}

\section{Discusión.}

Este trabajo comparte, desde el punto de vista metodológico, las mismas limitaciones y sesgos que un trabajo anterior elaborado por nosotros ${ }^{10}$, extensibles también a otros estudios similares ${ }^{25}{ }^{26}$ en los que se advierte de estos problemas metodológicos, de amplio espectro, pues la información se recoge por diferentes profesionales, no clínicos algunos de ellos, en condiciones de escasa o nula confidencialidad. En el caso de los clínicos, existen diferencias en formación, hábitos diagnósticos, orientación terapéutica, que condicionan el proceso de evaluación diagnóstica, manifestándose de forma más acusada en la atención a niños y adolescentes (falta de un lenguaje, evaluación y clasificación diagnóstica comunes) ${ }^{35}$. Otro factor de distorsión añadido es la ausencia de una codificación diagnóstica específica, en muchos de los casos, tras la primera entrevista de evaluación. Este aspecto afecta de manera importante a este estudio, pues los datos sobre el juicio diagnóstico de los pacientes nuevos se extraen del programa informático y no de la propia historia clínica.

A todos estos factores cabe añadir la especificidad de la atención a niños y adolescentes ${ }^{34}$ : el proceso de evaluación es más complejo, requiere la consulta de más fuentes (el niño, la familia, la escuela y, en ocasiones, otras instituciones como Servicios Sociales, Equipos de Orientación Educativos, instancias judiciales, etc.) y, por consiguiente, se produce una prolongación temporal del proceso 
ORIGINALES Y REVISIONES

de evaluación, limitando la posibilidad de una codificación diagnóstica definitiva en una primera entrevista.

Por último, una limitación importante a cualquier trabajo epidemiológico en los Servicios de Salud Mental de la Comunidad de Madrid lo constituye el programa informático que viene empleándose desde hace años (RAC).

A pesar de estas limitaciones, consideramos que los resultados que se comentan a continuación pueden ser válidos para los objetivos de este trabajo y equiparables a otros estudios llevados a cabo en el ámbito de funcionamiento de los servicios públicos de salud mental de distrito ${ }^{15} 27678$.

En la actividad asistencial del año 2004 en el SSM de Aranjuez, tal y como se indica en la figura 3, se observa una cifras de incidencia y prevalencia muy por encima de las tasas medias estimadas de trastornos mentales en la infancia y adolescencia en España ${ }^{31}$. Sin perjuicio de otras consideraciones, creemos que las características de la población del distrito, recogidas en la tabla $1^{21}$, pueden justificar las elevadas cifras asistenciales, ya que es una zona que absorbe personas jóvenes de otros distritos, implicando el crecimiento del grupo de edad más joven. En el resto de los distritos del Área 11 el número de casos nuevos menores de dieciocho años en el 2004 es variable, siendo Villaverde el que contabiliza mayor número de casos y el que supera a Aranjuez ${ }^{3629}$.

En relación al distrito de Aranjuez, es congruente la alta contribución al total de nuevos vistos en el año 2004, siendo casi la cuarta parte de los mismos, niños y adolescentes (sin contar la franja de quince a dieciocho años) (tabla 4).

En un trabajo anterior ${ }^{10}$ señalábamos el predominio de mujeres sobre varones en el total de nuevos atendidos en un año $(57,6 \% / 42,4 \%)$. Esta situación se invierte cuando se considera sólo la población de niños y adolescentes hasta quince años (44, 9\% / 55,09\%). Mientras que en la población general, ser mujer aparece como potencial factor de riesgo, en el caso de los niños y adolescentes, el factor de riesgo lo constituye el ser varón.

La figura 4 muestra una distribución por edad diferente a la hallada en estudios similares ${ }^{15}$, en los que el número de casos nuevos aumenta con la edad. En este estudio, sin distinguir sexo, se confirma un ascenso sólo en el grupo de cero a 12 años, encontrando un descenso en el número de casos a partir de esta edad. Esta distribución no nos sorprende, ya que los adolescentes muestran resistencias para acudir a salud mental, así como cierto grado de autonomía, frente a la autoridad de los padres, en el cuidado de su salud. Si tenemos en cuenta la variable sexo, se observa que a partir de los 13 años disminuyen los casos nuevos de varones y aumentan los de mujeres, si bien a los 17 años ambos géneros descienden conjuntamente. Podemos considerar estas diferencias en el contexto de la pubertad como factor de riesgo diferencial según el sexo, favorecedor de psicopatología en las mujeres y protector en los varones 241615353721312 . Este mayor riesgo en las mujeres parece asociarse con el estadío III de Tanner ${ }^{16}{ }^{13}$. 
En relación con los diagnósticos en función de la edad (tabla 5), observamos unas peculiaridades dentro de cada uno de los subgrupos etarios.

En el grupo de cero a seis años, destaca el diagnóstico "Perturbación de las emociones de la niñez y adolescencia", casi la cuarta parte de los casos. A continuación sigue la categoría de "Sin especificar" con un $16 \%$. El $11 \%$ de los casos suponen trastornos adaptativos. Los trastornos psicóticos suponen sólo el 1,23\%. Este resultado es compatible con las críticas que recibe la CIE - 9, que cuenta con unas categorías diagnósticas difusas y amplias. Por otra parte, la evaluación en niños en estas edades resulta compleja, pues la sintomatología tiene un carácter frecuentemente evolutivo, inespecífico, a menudo reversible y cronodependiente. Existe además una alta dependencia del entorno ${ }^{3435} 3940$.

En el grupo de siete a doce años se produce un aumento muy notable del diagnóstico "Reacción de adaptación" (26,97 \%), compatible con la aparición de estresores nuevos e intensos en esta etapa, como son la escolarización, la relación con los iguales, la separación de los padres, la exigencia socializadora en la familia, etc. ${ }^{40}$ Como tercer diagnóstico más prevalente en esta franja, encontramos el "Síndrome hipercinético", con cifras más elevadas que en la etapa anterior. Este dato es coherente con la aparición de la instancia educativa, cuyas normas ponen de manifiesto los comportamientos intrusivos de los niños hiperactivos ${ }^{16}$ 1535201938 . Otros cambios, comparando con la etapa anterior, son los descensos en los trastornos de la alimentación y el sueño, aunque se mantienen los problemas relacionados con el control de los esfínteres, tal y como se recoge en la literatura científica ${ }^{35}$. Finalmente, se quintuplica el porcentaje de sujetos en la categoría de "Trastornos neuróticos" (5,26\%) 161537234 .

En el grupo de trece a diecisiete años se mantiene la primacía de los trastornos de adaptación, incluso con una cifra superior a las anteriores. Esta etapa evolutiva, pubertad y adolescencia, supone cambios que desafían la capacidad de afrontamiento del adolescente, desde el ámbito escolar (cambio de ciclo), desde lo relacional (iguales, enfrentamiento generacional), desde lo psicológico (maduración, identidad psicosexual), desde lo biológico (especialmente en la mujer), etc. 1621323 .

En esta etapa se produce también una mayor diversidad en cuanto a las formas diagnósticas, disminuyendo el "Síndrome hipercinético" pero apareciendo "nuevos" diagnósticos como los abusos de alcohol y drogas, dependencia de tóxicos, trastornos de la personalidad y "perturbación de la conducta no clasificada en otra parte" $(8,64 \%)$. Cabe pensar que esta diversificación pueda deberse a la inclusión en estas nuevas categorías de sujetos hiperactivos no diagnosticados previamente como tales ${ }^{39}$.

La alimentación, que en la primera etapa alcanzaba un 7,4 \%, desapareciendo en la segunda franja, vuelve a aparecer con idéntido porcentaje, pero, 
evidentemente, con una naturaleza diferente, esto es, más vinculativa y relacional en el período preescolar y con un cariz de trastorno de conducta alimentaria adulto (anorexia y bulimia). Estas cifras son coherentes con las halladas en otros estudios, tanto en muestra clínica como en población general ${ }^{24163523 .}$

En contraste con la mayor variabilidad de esta franja, hay que reseñar la desaparición de algunas categorías, como los problemas relacionados con los esfínteres.

En cuanto a la categoría de "Sin especificar", se observa una disminución en el porcentaje de casos según aumenta la edad. Este dato podría tener que ver con la complejidad del diagnóstico en la primera infancia. En un trabajo anterior 10 se apreciaban cambios en esta categoría, en el sentido de aumentar en las etapas de mayor edad (por encima de los sesenta años). Es posible que esta variabilidad de "Sin especificar" pueda deberse a la menor exigencia ambiental sobre el sujeto en la primera infancia y en la ancianidad, lo cual dificultaría el diagnóstico, pues éste se efectúa muy a menudo sobre la base de la interferencia en las áreas académicas, laborales, familiares, sociales, etc. Respecto a los sujetos "Sin patología", se observa un curso parecido: mayor porcentaje en la primera infancia y con disminución en las otras dos etapas. Este dato nos remite de nuevo a la complejidad del diagnóstico en edades tempranas, ya comentado anteriormente ${ }^{34}$ 353931 .

En la franja de la adolescencia aparece por primera vez, con una baja frecuencia, la categoría de "Psicosis afectivas". Esto no significa que no existan las depresiones mayores en el período prepuberal, lo que pone de manifiesto la dificultad para reconocerlas como tales y diagnosticarlas, más allá del cajón de sastre de categorías como "perturbación de las emociones propias de la niñez y adolescencia"34181615228.

Respecto a la variable sexo (tabla 6), encontramos en los varones las siguientes categorías diagnósticas más frecuentes, a saber, "Reacción de adaptación" (24,28\%), "Perturbación de las emociones propias de la niñez y adolescencia" (16,76\%), "Sin especificar" $(16,76 \%)$ y, finalmente, el "Síndrome hipercinético" (15,03\%). Sólo estas cuatro categorías suponen el 72, $83 \%$ de todos los varones.

En las mujeres, las categorías más frecuentes son: "Reacción de adaptación" (21,28\%), "Perturbación de las emociones propias de la niñez y adolescencia" (19,86\%), "Sin especificar" $(10,64 \%)$ y, finalmente, "Sin patología" (7,8 $\%)$. Estas cuatro categorías constituyen el 59, $58 \%$ del total de casos de mujeres. Se observa una menor acumulación de los casos, en unas cuantas categorías, que en la distribución de los varones. Sin embargo, la distribución de los varones muestra más variabilidad, pues casi todas las categorías cuentan con casos. Esto es compatible con los datos epidemiológicos, que informan de un predominio de 
determinadas categorías en los varones frente a las mujeres, esto es, los trastornos del neurodesarrollo de inicio precoz (Trastorno generalizado del desarrollo, retraso mental, tics, etc.) ${ }^{18163537}$.

Si comparamos las diferentes categorías diagnosticas entre ambos sexos, hallamos los siguientes contrastes: en el "Síndrome hipercinético" la muestra ofrece un $15,03 \%$ de varones frente a un $4,26 \%$ de mujeres. Este contraste es refrendado en diferente estudios, tanto poblacionales como muestras clínicas ${ }^{34} 18$ 16153720193 . En el diagnóstico "Perturbación de la conducta no clasificada en otra parte" se invierte la proporción, existiendo en las mujeres un 6,38 \%, mientras que en los hombres sólo hay un $1,73 \%$. ¿Son estas diferencias explicables en función de prejuicios diagnósticos? Podemos especular que se tiende a diagnosticar el "Síndrome hiperactivo" en varones, mientras que quizá se subestime en mujeres. ¿Cabe pensar si estas diferencias obedecen a presentaciones clínicas dispares (mayor predominio de subtipo déficit de atención en niñas)? ¿Resultan igual de apropiados los criterios diagnósticos para los niños que para las niñas? En las mujeres la hiperactividad podría disfrazarse de trastorno de conducta, lo cual se reflejaría en la baja frecuencia de un diagnóstico y el alza del otro 16201938 9221 .

Hay que tener en cuenta que en este estudio sólo se tiene en cuenta el primer diagnóstico. Es conocida la asociación del "Síndrome hipercinético" con otros diagnósticos, particularmente con los "Trastornos de conducta", lo que podría explicar la menor presencia de "Perturbación de la conducta" en varones, al aparecer como segundo diagnóstico. Por otra parte la CIE-9 identifica la categoría "Trastorno hipercinético de la conducta" para aquellos casos que cumplen también criterios de "Síndrome hipercinético".

Otra diferencia tiene que ver con la predominancia del diagnóstico "Trastornos neuróticos" entre las mujeres, en comparación con los varones (6,38 / 2, 89), coherente con la literatura 1816153537 que señala una mayor frecuencia de trastornos emocionales en las mujeres frente a los varones.

Finalmente en este estudio se relacionan los diagnósticos en función de la edad y el sexo (tablas 7,8 y 9 ).

$\mathrm{Si}$ atendemos al grupo de edad de cero seis años, observamos (tabla 7), en contra de lo registrado en múltiples estudios, una predominancia de los trastornos del control de esfínteres en las mujeres. Podría ser debido al orden de codificación, y que en el caso de los varones las alteraciones de esfínteres ocuparan segundos y terceros puestos en cuanto al orden de los diagnósticos, suponiendo este dato, de ser así, una mayor comorbilidad en varones.

En esta franja, el diagnóstico más frecuente en ambos sexos es "Perturbación de las emociones propias de la niñez y adolescencia", pero en los varones constituye casi el $30 \%$ de los casos, mientras que en las mujeres sólo supone el 
ORIGINALES Y REVISIONES

$17 \%$. La proporción inversa la encontramos en las categorías "Reacción de adaptación" y "Reacción aguda ante gran tensión", usadas con más frecuencia en el diagnóstico de las mujeres. ¿Por qué se diagnostican más trastornos adaptativos en las niñas en la primera infancia? ¿Significan estas diferencias que operan más los factores individuales neurobiológicos (mayor vulnerabilidad del neurodesarrollo) en los varones y los contextuales en las mujeres en este grupo de edad? Hay que reparar en la ausencia de las categorías más "biológicas" en las mujeres, pues los trastornos del neurodesarrollo (Retraso mental, Retardo selectivo del desarrollo, Psicosis peculiares de la niñez) están ausentes, salvo en el caso de los esfínteres. En esta línea, reseñar la ausencia de casos de "síndrome hipercinético" en mujeres en esta franja, lo que viene a poner de relieve la predominancia de alteraciones del neurodesarrollo en varones ${ }^{18} 1637$.

Otra de las diferencias que suscita interrogantes es la existencia del diagnóstico "Trastornos neuróticos" en las mujeres, mientras que está ausente en varones. Esta ausencia podría estar compensada con el cajón de sastre de "Perturbación de las emociones de los niños y adolescentes" 1615 .

Finalmente, en esta franja de edad, los casos correspondientes al diagnóstico de "Psicosis peculiares de la niñez" corresponden todos ellos a varones, dato congruente con la literatura (6) ${ }^{34163337 .}$.

En el grupo de edad de siete a doce años (tabla 8) la categoría más diagnosticada y con cifras similares en ambos sexos es la "Reacción de adaptación", lo cual podría explicarse por el surgimiento de diferentes estresores en esta etapa (escolarización en Educación Primaria, exigencia de rendimiento, relación con iguales, etc.). En cuanto a las diferencias encontramos las siguientes:

En el "Síndrome Hipercinético" los varones vuelven a ser los predominantes $(18,88 \%$ del total de varones de esta franja). En la categoría "Sin especificar" el porcentaje de varones duplica al de mujeres, lo cual puede deberse a que el origen de la demanda tiene que ver con conductas perturbadoras, exigiendo por parte del profesional un proceso evaluativo más detallado (esto mismo se repite en la franja de los adolescentes). Otro motivo puede deberse a la elevada comorbilidad en los varones (Síndrome hipercinético asociado con trastornos del aprendizaje, trastornos de ansiedad, trastornos de conducta, etc.). En contraste, en la categoría de "No Patología" la situación se invierte, siendo casi insignificante el porcentaje de varones al que no se le encuentra patología en la primera entrevista. No resulta fácil contestar por qué hay más niñas "sanas" que niños entre las derivaciones de Atención Primaria fundamentalmente.

En la franja de la adolescencia (tabla 9) la "Reacción de adaptación" vuelve a ser lo más prevalente en ambas franjas, pero ya no hay cifras similares, predominando los varones. En la categoría de "Perturbación de las emociones propias de la niñez y adolescencia" el predominio es de la mujer. Ambos datos podrían 
ser complementarios, en el sentido de tender hacia el diagnóstico de lo adaptativo en varones y lo emocional en las mujeres, revelando la existencia de prejuicios de género. En esta línea, tenemos el diagnóstico de "Trastornos neuróticos” más frecuente también en las mujeres.

En "Trastornos de alimentación" más de un $13 \%$ de las mujeres de esta franja reciben un primer diagnóstico de este tipo, frente a la ausencia de esta categoría como primer diagnóstico en los varones ${ }^{24} 1816$. Además y/o en combinación con las presiones sociales y culturales, creemos que los cambios físicos en la mujer afectan más a su silueta que en el caso de los varones.

Los diagnósticos relacionados con el "Movimiento" (Tics fundamentalmente) ofrecen el resultado inverso, ausencia en mujeres y presencia en varones, congruente con lo mencionado anteriormente y con la literatura ${ }^{341637}$.

\section{Conclusiones.}

En este trabajo hemos expuesto las diferencias en el porcentaje de cada categoría diagnóstica en el total de nuevos menores de dieciocho años en función de las variables sexo, edad y la combinación de ambas.

Creemos que estas dos variables, aisladas o en combinación, suponen diferencias diagnósticas que deberían ser más investigadas, con el fin de aclarar etiologías, detectar factores de riesgo, identificar indicadores de cara a programas de tratamiento, prevención y rehabilitación, etc.

De entre todas las diferencias halladas en este Servicio de Salud Mental de distrito en función de ambas variables, nos parecen más relevantes las siguientes:

- Predominio de varones que consultan.

- La primera infancia podría constituir un factor de riesgo en los varones para los trastornos más graves (alteraciones del neurodesarrollo)

- La pubertad podría constituir un factor de riesgo para las mujeres y protector para los varones (disminuyen los casos de varones a partir de los 13 años)

- Ser varón es un factor de riesgo para el diagnóstico "Síndrome hipercinético" en cualquier franja de edad.

- Ser mujer podría ser un factor de riesgo para los diagnósticos comprendidos en la categoría "Trastornos neuróticos".

- Este trabajo confirma el dato repetido en muchos estudios de que ser mujer es un factor de riesgo, a partir de la pubertad, para los "Trastornos de alimentación"

- En la primera infancia los casos correspondientes al diagnóstico de "Psicosis peculiares de la niñez" corresponden todos a varones. 
ORIGINALES Y REVISIONES

- En ambos sexos la categoría más frecuente en la etapa de Educación Primaria y Secundaria es la de "Reacción de adaptación".

- La C. I. E. - 9 no es un instrumento diagnóstico apropiado, especialmente en la población infanto-juvenil, por la escasa definición de sus categorías diagnósticas.

- El programa informático Registro Acumulado de Casos de la Comunidad de Madrid no es un instrumento suficientemente fiable para la realización de estudios epidemiológicos, debido a su carácter obsoleto y a las limitaciones en los listados que proporciona. Resultaría adecuada su sustitución. 


\section{BIBLIOGRAFÍA:}

(1) AACAP, "Practice Parameters for the Assessment and Treatment of Children and Adolescents with Conduct Disorder", J. Am. Acad. Child and Adolesc. Psychiatry, October 1997 (Supplement), 36, 10.

(2) AACAP, "Practice Parameters for the Assessment and treatment of children and adolescents with Depressive Disorders", Journal of the American Academy of Child and Adolescent Psychiatry 1998, 37:63S-83S.

(3) ADHD EINAQ (European Interdisciplinary Network for ADHD Quality Assurance), Einaq and Thomson Advanced Therapeutics Communications, 2003

(4) AgüERo, JuAn A., "Epidemiología de los trastornos de Ansiedad", en MARDOMINGO SANZ, M. J. (Ed.), Trastornos de Ansiedad en el Niño y Adolescente, Smithkline-Beecham, Madrid 1999, p. 15-20.

(5) Angold, A, Costello, E. J., "Epidemiología del desarrollo: la naturaleza del riesgo en los trastornos psiquiátricos”, en EZPELETA, L. (Ed.), Factores de riesgo en psicopatología del desarrollo, Ed. Masson, Barcelona 2005, Pags 21-50

(6) Ballester Pérez C, y otros, "Análisis de la población atendida en la USMI II de Castellón 2004”, Póster presentado en la XLII Reunión de la AEPIJ, Bilbao 31 Mayo-2 Junio 2007, Publicado en la Revista de Psiquiatría infanto-juvenil 2007, Volumen 24, Número 1 (especial), Pags 142-143.

(7) Ballester Pérez C, y otros, "Estudio descriptivo de la incidencia en la población atendida en la USMI de Castellón 2005", Poster presentado en la XLII Reunión de la AEPIJ, Bilbao 31 Mayo-2 Junio 2007, Publicado en la Revista de Psiquiatría infanto-juvenil, 2007, Volumen 24, Número 1 (especial), Págs. 140-141.

(8) Ballester Pérez C, “Análisis comparativo de los nuevos casos de población infantojuvenil atendida durante los años 2004-2005 en la USMI II de Castellón”, Poster presentado en la XLII Reunión de la AEPIJ, Bilbao 31 Mayo-2 Junio 2007, Publicado en la Revista de Psiquiatría infanto-juvenil, 2007, Volumen 24, Número 1 (especial), Págs. 141-142.

(9) BaRkLEy R.A., Attention Deficit hiperactivity disorder: A handbook for diagnosis and treatment ( $3^{\mathrm{a}}$ Ed.), New York: Guilford Publications, 2006.

(10) Belloso Ropero, Ju, Espín Jaime, JC., "Un año de pacientes nuevos en un Servicio de Salud Mental”, Rev. Asoc. Esp. Neuropsiq., 2007, Vol. XXVII, no 99, pp. 39-58.

(11) Comunidad De Madrid, Consejería De Sanidad Y Servicios Sociales, Programas de los Servicios de Salud Mental, Madrid, Publicaciones del Servicio Regional de Salud, 1989.

(12) Dirección General De Farmacia Y Productos Sanitarios, Consejería de Sanidad y Consumo, Comunidad de Madrid, Recomendaciones para la valoración y tratamiento de la depresión infanto-juvenil, Recomendaciones Farmacoterapeúticas en Salud Mental, Febrero, 2006, № 3.

(13) Doménech-Llaberia E., "Epidemiología de la depresión y características especiales en la ado lescencia", En MARDOMINGO SANZ, M. J., (Ed.) II Jornadas de psiquiatría del niño y del adolescente, Estados Depresivos en la Adolescencia, Aportaciones para la práctica clínica, Smithkline-Beecham, Madrid 1997, p. 31-41.

(14) ElPERs, J. R., “Community Psychiatry”, En KAPLAN Y SADOCK, (Ed.), Comprehensive Textbook of Psychiatry, Sexta edición, Baltimore, Williams and Wilkins, 1995. 
(15) Espezel Olivella, M.I, y otros, "Análisis descriptivo de la población infanto-juvenil atendida en un Centro de Salud Mental Infanto-Juvenil comarcal”, Psiquiatría Biológica, Volumen 13, Extraordinario 3, Octubre 2006, Págs. 109-110, Relativo al X Congreso Nacional de Psiquiatría, Sevilla, 16-21 Octubre de 2006.

(16) EzPeleta, L., "Género y psicopatología”, . En EZPELETA, L. (Ed.), Factores de riesgo en psicopatología del desarrollo, Ed. Masson, Barcelona 2005, Págs. 79-111.

(17) Fabiano, G.A, Pelham JR, W.E., "Evidence-based treatment for Mental Disorders in children and adolescents", Current Psychiatry Reports, Vol. 4, Number 2, April 2002, Págs. 93-100.

(18) Franco, C, Arango, C., "Prevalencia de Trastornos mentales en niños y adolescentes", Monografías de Psiquiatría, Vol. XVI, № 3, Julio-Septiembre 2004, Págs. 19-27.

(19) García Giral, M., Nicolau Palou, R., "Actualizaciones en el tratamiento del TDAH", Aula Médica Psiquiátrica, N 3, 2001, Págs. 180-214.

(20) García M, Nicolau R, Toro D, Roses R, Cabrera B., "El TDAH: diferencias según género", Póster presentado en la XLII Reunión de la AEPIJ, Bilbao 31 Mayo-2 Junio 2007, Publicado en la Revista de Psiquiatría infanto-juvenil, Volumen 24, Número 1 (especial), 2007, p. 151.

(21) Instituto De Estadística De La Comunidad De Madrid, Reseña estadística zonal de 2005, Madrid, Comunidad de Madrid, 2005.

(22) Loeber, R. Y Otros, "Oppositional Defiant and Conduct Disorder: A Review of the Past 10 Years, Part I.", J. Am. Acad. Child Adolesc. Psychiatry, December, 2000, 39: 12.

(23) Lozano Oyola Jose F., Rodríguez Sacristán J., "La psicopatología en la edad escolar y en la adolescencia”, En RODRÍGUEZ SACRISTÁN, J. (Ed.), Psicopatología Infantil Básica, Teoría y Casos Clínicos, Psicología Pirámide, Madrid 2000, Págs. 271-286

(24) Mardomingo Sanz, M. J., "Trastornos psiquiátricos de la infancia y adolescencia: Avances recientes y previsión para el futuro", Monografías de Psiquiatría, X Aniversario, Vol. XI. № 5. Septiembre-Diciembre 1999, Págs. 38-40.

(25) Martínez, O. Y Otros, "Estudio de las primeras consultas de un centro de Salud Mental (I), Características sociodemográficas, vías de derivación y tratamiento anterior", Rev. Asoc. Esp. Neuropsiq., 1997, Vol. XVII, nº 61, Págs. 37-60.

(26) Montilla, J. F. Y Otros, "Uso de servicios ambulatorios de Salud Mental en España, Consumo de recursos en el primer año de asistencia a pacientes nuevos", Rev. Asoc. Esp. Neuropsiq., 2002, Vol. XXI, nº 84, pp. $25-47$.

(27) Ochoa, S, Linares, M.D., "Evolución de la demanda de AP a SMIJ", Revista de Psiquiatría Infanto-Juvenil Número 2/2003, Abril-Junio, Págs. 66-69.

(28) OFFord, D.R., "Epidemiología de los trastornos psiquiátricos en la infancia y la adolescencia”, en GELDER, M.G, LÓPEZ-IBOR JR, J.J, ANDREASEN, N. Tratado de Psiquiatría, Tomo III, Ars Médica, Barcelona 2003, Págs. 2032-2037

(29) ORCSM, Análisis del Registro de Casos Psiquiátricos de la Comunidad de Madrid, Años 1995-2004, Análisis preliminar, Comunidad de Madrid: Febrero, 2007.

(30) Organización Mundial De La Salud, Clasificación Estadística Internacional de las Enfermedades, Lesiones y Causas de Muerte, Novena revisión, Ginebra, O. M. S., 1977.

(31) Pedreira Massa, J. L., "Dilemas actuales y perspectivas de la psiquiatría de la infancia y la adolescencia", Monografías de Psiquiatría: Psiquiatría Infanto - Juvenil (I): Psicopatología, X aniversario, Año XI, enero - febrero 1999, nº 1, pp. 1-6. 
(32) Roberts, R.E., y otros, "Prevalence of Psychopathology Among Children and Adolescents", Am J. Psychiatry 155, 6, June, 1998, Págs. 715-725.

(33) RutTer, M., "Development and psychopathology", En RUTTER, M., TAYLOR, E., (Eds.), Child and adolescent psychiatry, Oxford, Ed. Blackwell, 2002.

(34) San Sebastián Cabases, J., "Psiquiatría de la infancia y adolescencia, Parte III Problemas clínicos emergentes", en López-Ibor Aliño JJ, Gómez Pérez JC, Gutierrez Fuentes JA (Eds.), Retos para la Psiquiatría y la Salud Mental en España, Ars Médica, Barcelona 2003, págs. 187-204.

(35) San Sebastián Cabases, J., "Situación actual de la asistencia en Salud Mental de niños y adolescentes en la Comunidad Autónoma de Madrid", Estudios e Investigaciones, 2002, Págs. 235260.

(36) Servicio De Psiquiatría Del H. G. U. 12 De Octubre De Madrid, Memorias de los SSM del área 11, Documentación interna de área.

(37) Sсотt, S., "Psicopatología del desarrollo y clasificación en la infancia y la adolescencia”, en GELDER, M.G, LÓPEZ-IBOR JR, J.J, ANDREASEN, N., Tratado de Psiquiatría, Tomo III, Ars Médica, Barcelona 2003, Págs. 2019-2025.

(38) Scott, S., "Trastornos del comportamiento en la infancia y adolescencia", en: GELDER, M.G, LÓPEZ-IBOR JR, J.J, ANDREASEN, N. Tratado de Psiquiatría, Tomo III, Ars Médica, Barcelona 2003, Pags. 2097-2111

(39) Torres Iglesias A, Domínguez S antos M. ${ }^{a}$ D. Y Rodríguez Sacristán J., "La Psiquiatría Infantil, hoy: (II) Principales Fundamentos Teóricos, Avances en Salud Mental Relacional”, Revista Internacional On-line, Vol. 3, núm. 3. Noviembre 2004.

(40) Torres Iglesias A, Domínguez Santos M M D Y Rodríguez Sacristán J., "La Psiquiatría Infantil, hoy:(III) Concepto, Objeto de estudio y Método, Avances en Salud Mental Relacional", Revista Internacional On-line, Vol. 3, n ${ }^{\circ}$ 1, marzo 2004.

* José Carlos Espín Jaime. F. E. A. Psiquiatría. Unidad de Psiquiatría Infantojuvenil de Interconsulta - Enlace. Servicio de Psiquiatría. H. U. 12 de octubre. Avda. Córdoba s /n. 28041. Madrid. Juan José Belloso Ropero. F. E. A. Psicólogo Clínico. Programa de Trastornos Mentales Graves. Servicio de Psiquiatría. H. U. 12 de octubre. Avda. Córdoba s /n. 28041 Madrid.

Correspondencia: José Carlos Espín Jaime. Correo electrónico: jcespinj@telefonica.net

** Recibido: 27/10/2008 